\title{
Modelo de gestión de equipos virtuales en la ejecución de proyectos académicos colaborativos *
}

\author{
Virtual team management model for executing collaborative academic \\ projects
}

\author{
Nicolás Borrero-Puentes \\ Estudiante de Ingeniería industrial, Universidad Santo Tomas, \\ Bogotá, Colombia, nicolasborrero@gmail.com \\ Luisa Fernanda Alcalá-Zarate \\ Magíster en Ingeniería Administrativa e Innovación, Universidad Santo Tomas, \\ Bogotá, Colombia, luisaalcala@usantotomas.edu.co
}

\begin{abstract}
Cómo citar / How to cite
Borrero-Puentes, N.; Alcalá-Zarate, L. F. (2020). Modelo de gestión de equipos virtuales en la ejecución de proyectos académicos colaborativos. Revista CEA, v. 6, n. 12, 147-166.

https://doi.org/10.22430/24223182.1609
\end{abstract}

Recibido: 2 de marzo de 2020

Aceptado: 28 de mayo de 2020

\section{Resumen}

Al estar inmersos en la era del conocimiento, es de vital importancia que los profesionales, en especial los ingenieros, tengan una formación integral donde la academia sea el principal actor que proponga actividades que permitan actuar y pensar globalmente, pues esto será la clave del crecimiento de las naciones y de la industria local. Por tal motivo, esta investigación buscó desarrollar un modelo de gestión de equipos virtuales que permitió ejecutar proyectos académicos colaborativos entre la Universidad Santo Tomás (USTA) y el Tecnológico de Costa Rica (TEC). Para ello se hizo un prepilotaje que dio a conocer las variables más importantes en este tipo de ejercicios, de las cuales destacaron la comunicación, el tiempo, el trabajo colaborativo y el manejo de las TIC. Seguidamente, se hizo una búsqueda en bases de datos como Scopus, Web Of Science y Google Académico, y se encontró que las metodologías ágiles y tradicionales en proyectos son necesarias para la estructuración y flexibilidad del mismo. Finalmente, se identificó que los individuos, los procesos y la tecnología gestan nuevos conocimientos que benefician una comunidad en concreto, potenciando así sus habilidades

\footnotetext{
* Este artículo se deriva del proyecto titulado «Modelo de gestión de equipos virtuales para la ejecución de proyectos académicos colaborativos caso de estudio Universidad Santo Tomás y el Tecnológico de Costa Rica» y ha sido financiado con recursos de ambas instituciones.
} 
y transformando debilidades. De este modo, la vinculación de estos espacios en la academia hizo que los estudiantes desarrollaran una autonomía en sus procesos de aprendizaje, adquiriendo metodologías que serán de gran utilidad en la vida laboral. Adicionalmente, se pudo simular el ambiente real que viven las empresas transnacionales, lo que ayudó a consolidar y generar un panorama holístico de las actividades que realizan. Lo anterior favorece a cada uno de los involucrados, pues sus conocimientos se vieron fuertemente potenciados, y responde al problema que existe entre lo teórico y la praxis, donde existe una gran brecha que cerrar.

Palabras clave: modelo educacional, cooperación educacional, entornos virtuales de aprendizaje.

Clasificación JEL: 129, M19, M54.

\section{Abstract}

Since we are immersed in the Knowledge Age, professionals (especially engineers) should receive a comprehensive training in which the academia becomes the main actor proposing activities that allow them to act and think globally because this will be key to the growth of nations and the local industry. For this reason, this study sought to develop a virtual team management model that allowed Universidad Santo Tomás (USTA) and Tecnológico de Costa Rica (TEC) to execute collaborative academic projects with each other. For this purpose, we conducted a pre-pilot study that revealed the most important variables for such activities, including communication, time, teamwork, and ICT use. Then, after a literature review in major databases, such as Scopus, Web of Science, and Google Scholar, we found that agile and traditional project management methodologies are necessary for the shaping and flexibility of projects. Finally, we realized that individuals, processes, and technology generate new knowledge that benefits a specific community, thus enhancing their abilities and transforming their weaknesses into opportunities. Therefore, incorporating these spaces into the academia helps students develop autonomy in their learning processes and learn methodologies that will be very useful in their working life. Additionally, we were able to simulate the real context of transnational companies, which served to consolidate and create a holistic overview of the activities they perform. This favors each party involved because their knowledge is strongly enhanced. In addition, this addresses the current problem between theory and praxis, where there is still a great gap that needs to be bridged.

Keywords: Educational models, educational cooperation, virtual learning environments.

JEL classification: 129, M19, M54.

\section{INTRODUCCIÓN}

La competitividad se refiere a una rivalidad constante para conseguir un fin (Pérez Porto \& Merino, 2016; Teagarden, 2020). Este término es muy usado por las empresas, ya que, a lo largo de sus operaciones, se esfuerzan cada vez por ser más fuertes y diferenciadoras en el mercado actual (Acevedo-Prins \& Jiménez-Gómez, 2015; Gupta et al., 2020). Es por ello que implementan diversas técnicas, conceptos, principios teóricos y metodologías que les permitan ser referentes locales y globales. De manera contradictoria, estas no prestan atención a la gestión del conocimiento, que 
supone un factor clave para adquirir y generar valor en cada uno de los actores principales, como las firmas, los empresarios y colaboradores (Antunes \& Pinheiro, 2019; Acevedo-Correa et al., 2020). Al identificar, filtrar, seleccionar y transmitir información, se genera un proceso de innovación organizacional y personal, debido a un discernimiento y comprensión del entorno como la teoría y las experiencias propias de cada industria (Barros et al., 2020; Jasso Villazul \& Torres Vargas, 2008). En este sentido, son pocas las compañías que exploran en la interacción con otras culturas a través de la ejecución de proyectos virtuales, donde los involucrados fortalecen diversas habilidades que les permitan ser más productivos y efectivos al momento de satisfacer y atender las necesidades de los clientes.

A este respecto, los profesionales actuales están sufriendo un fenómeno que afecta su iniciativa y capacidad de generar propuestas innovadoras que cambien la forma de ejecutar los procesos en las organizaciones. Dado este problema, es posible cuestionarse de qué forma enseñan las instituciones educativas hoy en día y si actúan en función del mundo globalizado. De igual manera, cabría preguntarse lo siguiente: ¿Los docentes realmente trabajan en modo colaborativo? ¿Los estudiantes se preocupan por entender el entorno en el que viven? ¿Los profesionales fueron formados bajo el principio de esta nueva era del conocimiento? ¿Los ingenieros industriales latinoamericanos entienden que es la industria 4?0, la globalización y la conectividad? (Hernández Cotón \& Sánchez Gutiérrez, 2006; Martínez Villalobos et al., 2016). Las anteriores incógnitas permiten identificar el nivel en el que se encuentran hoy en día y la actitud y postura de todos los stakeholders que involucran la educación actualmente.

A raíz de la situación anteriormente planteada, surge la presente investigación, en la que se considera necesario ahondar en soluciones prácticas que permitan la implementación de grupos virtuales en la academia para la ejecución de proyectos a distancia, en donde se conforme una sinergia junto con el trabajo colaborativo para aumentar y potencializar los conocimientos personales y grupales de un entorno en específico. Ahora bien, forjar estos espacios en las instituciones educativas crea en los actores directos una visión general de la forma en que operan las empresas transnacionales. Así, se tendrá una herramienta para aportar grandes ideas y material significativo a las organizaciones a las cuales pertenezca, generando así nuevos conocimientos claves para el desarrollo y crecimiento de las firmas.

En la presente investigación, que se lleva a cabo en conjunto con el Tecnológico de Costa Rica (TEC) y la Universidad Santo Tomás de Colombia (USTA) en la Facultad de Ingeniería de Producción Industrial e Ingeniería Industrial, respectivamente, se simula un ambiente productivo real con el fin de llevar a cabo un ejercicio práctico que relacione un espacio académico como Procesos Industriales. Con este fin, se integra una metodología de trabajo colaborativo que permita identificar las relaciones interpersonales, comunicativas y transferencia de conocimiento, logrando así la formación integral del ingeniero industrial, profesional que evalúe, mida y consolide cada una de las habilidades anteriormente mencionadas. Su estrategia de acción gira en torno al diseño virtual de un modelo de gestión de equipos que faculte al estudiante y al docente para estructurar, desarrollar, interactuar e involucrarse con personas de distintas zonas geográficas. De este modo, adquieren competencias holísticas en la resolución de problemas, liderazgo en proyectos, toma de decisiones y coordinación con otros a la distancia. 
En este sentido, ¿qué elementos debe considerar un modelo de gestión de equipos virtuales en la ejecución de proyectos académicos colaborativos en el estudio de caso de la Universidad Santo Tomás y el Tecnológico de Costa Rica? Para poder responder esta pregunta se debió elaborar un estado del arte de las metodologías y herramientas existentes para evaluar la gestión de equipos virtuales que intervienen en la gerencia de proyectos, lo que dio paso a diseñar el modelo Segev en la ejecución de proyectos académicos a través del trabajo colaborativo.

\section{METODOLOGÍA}

El tipo de estudio que se empleó corresponde a un estudio no experimental de corte transversal y de carácter descriptivo, que pretendió puntualizar en los elementos que se debieron considerar para la gestión de equipos virtuales en la ejecución de proyectos académicos colaborativos y sin manipulación de las variables de estudio. El método de muestra tuvo un carácter no probabilísticomuestreo, por conveniencia, para recopilar información relevante en el corto plazo.

Las técnicas y métodos para la recolección y análisis de información consistieron, inicialmente, en un análisis bibliométrico de bases datos por medio de búsqueda sistemática y lectura comparada. Se consolidó una base de conocimiento en la definición del modelo de gestión de equipos virtuales y ejecución de proyectos académicos colaborativos entre las dos instituciones. Posteriormente, se analizaron y depuraron las variables más relevantes identificadas en los modelos de gestión del conocimiento, gerencia de proyectos y trabajo colaborativo, generando así mapas mentales que permitieron la simplificación de información. Finalmente, se llevó a cabo un prepiloto simulando la ejecución del proyecto académico en el que se implementó la técnica de la observación directa y la aplicación de cuestionarios como herramienta para la definición de criterios incluyentes y excluyentes en la gestión de equipos virtuales.

Una vez identificadas las variables incluyentes y excluyentes de cada artículo, proyecto y metodología analizada, se realizó una sesión de evaluación por cuenta de expertos en temas de gestión del conocimiento, gerencia de proyectos y trabajo colaborativo. Ellos dieron su aval para implementar o no cada criterio y herramienta a vincular en el modelo de gestión de equipos virtuales.

\section{Procedimiento}

Para proceder, fueron requeridos los siguientes pasos:

1. Realizar un prepilotaje que permitiera suministrar información de primera mano, evidenciando los puntos de fluctuación que debieran ser controlados.

2. Analizar la literatura en cuanto a las características que debe considerar un modelo de gestión de equipos virtuales y el desempeño de sus actividades. Se elaboró una fórmula de búsqueda que relacionaba un eje temático junto con una palabra clave y el formato del documento, por ejemplo: «Gerencia de Proyectos» Scrum: filetype:pdf7.

3. Identificar los elementos clave para la planeación y ejecución del modelo, teniendo en cuenta la conformación de los equipos, la comunicación, el tiempo permisible, entre otros. Para ello se 
construyó una matriz que relacionaba al eje temático, el tipo de investigación, el tipo de proyecto, las ciudades o países que lo abordaron y la importancia de las variables.

4. Diseñar el instrumento para abordar la gestión de equipos virtuales en la ejecución de proyectos académicos colaborativos. Luego del análisis de literatura y de seleccionar las variables más importantes de los modelos de equipos virtuales, se conformó el modelo Segev, teniendo claro que fuese secuencial, sencillo de entender, visualmente agradable y técnicamente efectivo.

5. Diseñar la guía operativa de la manera en la cual se debe implementar el modelo en las aulas de clase. En este paso se desarrolló un documento externo, en el que secuencialmente se describía cada uno de los pasos a ejecutar para implementar el modelo Segev, y se resume en un diagrama de flujo que permite la simplificación de cada una de las fases.

6. Validar el modelo bajo el juicio de expertos referido a cada concepto clave identificado. Para ello, fue necesario desarrollar un cuestionario en el que, por medio de preguntas persuasivas, cada uno de los expertos valoraba los componentes o ejes temáticos del modelo. Algunas de las preguntas fueron: ¿La metodología planteada bajo la configuración del modelo Segev es aplicable en la Universidad Santo Tomás y el Tecnológico de Costa Rica? Y, ¿̇las herramientas fueron pertinentes y flexibles para alcanzar los objetivos?

\section{RESULTADOS}

En la creación del modelo propuesto como primera parte, se realiza un prepilotaje en el que se ejecuta un proyecto del espacio académico de Producción Industrial, diseñando y manufacturando un carro Jeep, empleando el software CAD Fusión 360 y la manipulación de las impresoras 3D y láser. En este sentido, cabe resaltar que hubo aciertos, falencias y oportunidades de mejora en el prepilotaje del ejercicio. Entre los aciertos destacan el cumplimiento de manufactura y diseño del producto (donde surgen bastantes oportunidades de mejora), la apertura a nuevas relaciones personales entre los estudiantes y docentes y el buen manejo de las tecnologías de la información.

En cuanto a las falencias se encontró poca comunicación entre los académicos, la inexistencia de las metodologías que permiten estructurar y desarrollar el proyecto a distancia, la falta de contemplación de los estándares de calidad para la mitigación de los riesgos tanto del proyecto como del producto. Asimismo, tampoco se estableció una asignación clara de roles, ni del alcance de estos con un cronograma que trazara el camino de las reuniones, lo que ocasionó retrasos en la planificación, ejecución, control y finalización del proyecto, por lo que la entrega de las actividades se hizo a destiempo y en formatos diferentes.

En las oportunidades de mejora, se percibe la definición de un nivel de formalismo del chat, junto con un formato de entrega de documentos, las posibilidades de alianzas con empresas y otras facultades, la elaboración de un protocolo de solución de conflictos y la asignación de sesiones de bocetos a mano y en línea.

Para cada una de las etapas de un proyecto se fijaron ciertos análisis que responden a cuestionamientos de cada una de ellas; en la fase de inicio surgieron los siguientes: ¿Se consideraron 
los beneficios de realizar el proyecto? ¿Es viable el plan? En planificación: ¿Se tuvo en cuenta la triple restricción de un proyecto? ¿Se analizó cada área del conocimiento para los proyectos? En ejecución: ¿Se utilizaron los equipos correctos? ¿Se manufacturó y ensambló en los tiempos acordados? En control: ¿Se realizaron ajustes o correcciones al producto fabricado? ¿Qué indicadores de rendimiento se usaron para medir el desempeño del proyecto? Finalmente, en el cierre: ¿Se comunicó la finalización del proyecto? ¿Se documentó el trabajo realizado? En razón a lo anterior, la valoración porcentual de estas cinco etapas tuvo un $23.4 \% 12.5 \%, 100 \%, 100 \%$, $90 \%$ de favorabilidad respectivamente.

Por otro lado, se realizó un seguimiento semanal al comportamiento comunicativo e interactivo de los grupos tanto de Colombia como de Costa Rica, en el que se logró concluir que de los diez miembros involucrados en el ejercicio y con un total de nueve reuniones virtuales, asistían en promedio dos y tres estudiantes de cada instituto educativo respectivamente, lo que da como resultado una relación entre sí del 50\%. A raíz del horizonte de tiempo empleado en el prepiloto, el cual fue de quince semanas, hubo una señal de participación y difusión de la información en tan solo nueve semanas, donde se evidenció la selección, almacenamiento, distribución y uso de la misma, dejando entonces una relevancia del $60 \%$ de comunicación en el ejercicio sostenido.

Adicionalmente, se requirió complementar la creación del modelo de equipos virtuales con tres temas claves, que debían tener una relación entre sí y contribuir a la ejecución de proyectos académicos a distancia. Estos fueron:

1. Trabajo colaborativo. En respuesta a la metodología usada, se realizó una revisión exhaustiva en las bases de datos Scopus, Web Of Science y Google Académico. Se analizaron técnicas y artículos que plantearan las ventajas y desventajas de las técnicas cooperativas, debido a que fue una buena estrategia para aumentar el conocimiento en una población en específico (Mora-Vicarioli \& Hooper-Simpson, 2016), por lo tanto, se presentarán cada una de las siguientes herramientas:

a) Los nueve roles de equipo. Esta herramienta pretende conformar equipos de alto rendimiento, a través de la aplicación de un instructivo que clasifica al individuo en roles mentales, sociales y de acción (Belbin, 2014). Debido a su gran fortaleza, es apto y diseñado para conformar grupos multidisciplinares.

b) Las habilidades de un equipo. Dicha herramienta fue construida para que cada integrante sea libre de tomar sus propias decisiones, además, esta técnica divide a los miembros del grupo en tres clasificaciones rol de negocios, emocional y vital (Peña, 2016). Su falencia consiste en que solo funciona con grupos de una misma disciplina.

c) Las TIC. Esta metodología ayuda a que los miembros de los equipos puedan modelar, comunicar y difundir información en diferentes puntos geográficos, pues adopta las herramientas Cloud computing, que permiten concentrar todo el conocimiento en carpetas compartidas en la nube. También posibilita a los individuos a realizar reuniones online, situación que genera flexibilidad en la comunicación y gestión de proyecto (Ángel Uribe \& Cano Vásquez, 2011).

En consecuencia, en la síntesis e interpretación de múltiples artículos de investigaciones avanzadas se logran identificar aspectos importantes que son útiles de considerar como el trabajo colaborativo. 
Este es un proceso en el que cada integrante adquiere información y aprende más de lo que podría conseguir él solo, resultado de la interacción entre los miembros del equipo (Podestá, 2014). También, la conformación de equipos de alto desempeño está ligada directamente al trabajo colaborativo que se geste durante la selección y conformación de los mismos (Belbin, 2015; Sung \& Choi, 2012), por lo que es obligatoria la asignación de los trabajos a cada uno de los miembros en relación con sus competencias, conocimientos y habilidades (Rugeles Contreras et al., 2015). Se debe hacer partícipe al estudiante en su proceso de aprendizaje, en el que se vuelva miembro activo y responsable de su aumento intelectual, proponiendo dinámicas que favorezcan la ejecución de proyectos en los que prime el aprender interactuando y compartiendo (Apolo Peñaloza \& Hidalgo Ochoa, 2016). En esa medida, el trabajo colaborativo cobró sentido en tanto que se eliminan las distancias físicas, existe una flexibilidad horaria, hay presencia de metodologías de asignación de responsabilidades basados en sus competencias y existe una resolución instantánea a los problemas (Vaillant, 2016).

Finalmente, se obtuvieron características generales de la ejecución de un trabajo colaborativo en entornos de proyectos a distancia, estos son: manejo de las habilidades comunicativas, asignación de tareas según sus conocimientos, responsabilidad individual recíproca, motivación por alcanzar el objetivo trazado y establecimiento de relaciones simétricas (Guitert \& Giménez, 2000).

Una vez conocido el perfil activo de cada integrante, se realiza una asignación de las tareas dependiendo de sus capacidades y competencias, en las que destaca una metodología colaborativa que logre plasmar las habilidades de los integrantes. Por esto, es necesario evaluar el instrumento que más se ajuste al ejercicio (Pinzón Rincon \& Remolina Millán, 2017), entre los cuales está la matriz raci, que permite visualizar claramente el rol de cada persona y la responsabilidad con respecto a cada entregable. Esta matriz es dinámica y flexible, y es ideal para distinguir las labores entre los operarios (Cavaller et al., 2014). También se encuentra la Job Description, que, como su nombre lo dice, es la descripción del puesto de trabajo sesgado a una actividad específica en la que se exponen las principales competencias y entregables (De la Cruz Lablanca, 2015). Finalmente, los Tableros Visuales, que se asimilan a la metodología Kanban, solo que, en lugar de colocar el nombre de las actividades, se coloca el nombre del responsable, y en la matriz se asignan micro objetivos que están enlazados con los entregables generales (Estrada Sentí, 2014).

Con los anteriores hallazgos, es necesario identificar una herramienta o técnica que cumpliese con criterios de inclusión y exclusión para su posterior articulación al modelo generado. En cuanto a criterios de inclusión, están las herramientas usadas que deben de ser de sencilla de manipulación, cuya técnica debe tener un componente dinámico en el que la asignación del entregable sea flexible y se debe de ajustar la coordinación entre varias áreas organizativas o grupos de trabajo (Ángel Uribe \& Cano Vásquez, 2011). Para los criterios de exclusión, las especificaciones de las competencias o habilidades no son claras, por lo que cada uno de los integrantes del equipo debe dejar en evidencia cuáles han sido sus competencias fuertes y blandas en la experiencia en la ejecución de proyectos; de no ser así, la herramienta no es funcional. Tampoco se identificaron buenas prácticas, ya que el individuo debe de explicar cómo es su sistema de almacenamiento y difusión de la información y, finalmente, se halla la descripción de sus debilidades, porque, al contrario del primer ítem, el integrante debe ser claro en cuáles son sus falencias dentro de la ejecución de proyectos a distancia, para que sea facilitada o corregida por herramientas de trasmisión de la información. 
En cuanto a la gerencia de proyectos, gracias a la información con la que cuentan las bases de datos Scopus, Web Of Science y Google Académico se logran analizar diversos trabajos virtuales en los que se identifican metodologías de gerencia de proyectos tradicionales y ágiles que ayudan a estructurar y reducir el riesgo, tornándolos flexibles (Bates \& Sangra, 2012). En razón a esto, resulta útil implementarlas en la ejecución de proyectos académicos virtuales; en este sentido, se mostrarán las herramientas que se analizaron para la vinculación de las mismas al modelo que se propuso, explicando su funcionamiento y principales ventajas y desventajas.

El texto pmbok es una metodología que permite estructurar un proyecto haciendo énfasis en las áreas del conocimiento y las fases del mismo. En esta se hace una exhaustiva previsibilidad de los fallos, planteando procedimientos que ayuden a controlar, mitigar o reducir el impacto; es de gran utilidad en un ambiente en el que se quiera tener todo bajo vigilancia. Esta técnica es la más usada a nivel mundial, pues es muy completa a la hora de liderar un proyecto en una compañía; su desventaja radica en que es una metodología tradicional y el aporte del cliente no es igual de fuerte que en las metodologías ágiles (Cuatrecasas Arbós, 2012).

La herramienta Scrum sirve para administrar y reducir el riesgo de los entregables, minimizando también el tiempo de duración de las actividades (Addati, 2017), pues en esta se estipula que el límite para la finalización de cada tarea debe tener un máximo de cuatro semanas. Está diseñada principalmente para adaptarse a los cambios fortuitos del entorno, traducido en los requerimientos del cliente debido a la influencia de un mercado competitivo; destaca su inspección periódica y la autogestión e innovación (Amaro Calderón \& Valverde Rebaza, 2007). Adicionalmente, se ve reflejada en plataformas online que arrojan datos del progreso del proyecto en tiempo real como Monday, que adoptó dicha metodología en su servicio.

La herramienta Iconix sobresale en proyectos informáticos por su fuerte enfoque en el seguimiento de los requisitos codificados en plataformas online; utiliza en gran medida los diagramas uml y se apalanca del rup, que es la base del conocimiento para poder sintetizar la información dentro del sistema Iconix, pues permite analizar y documentar cualquier tipo de sistema (Lui et al., 2020).

La metodología Extreme programming destaca por ser altamente adaptable a los requerimientos, optimizando los costos más que por la estructura y visualización de los riesgos o de la calidad con la que tiene que responder el producto o servicio. Se caracteriza, entonces, por un desarrollo interactivo e incremental, validaciones continuas y frecuentes correcciones en los requerimientos iniciales (Beck, 1999).

Finalmente, la herramienta de comunicación en tiempo real Kanban es utilizada para controlar el avance del trabajo en una línea de producción, simplificando la planificación y la asignación de responsabilidades tabuladas en un workflow. Posee una desventaja, que no es apta para grandes aumentos de la demanda, pues sus actividades están delimitadas al día de trabajo (Gilibets, 2013).

En este orden de ideas, se presenta a continuación la Tabla 1, que resume los criterios de Inclusión y de Exclusión para la selección de las metodologías, herramientas y modelos de proyectos que permitan dar una pronta respuesta con respecto a los requerimientos del cliente. 
Tabla 1. Síntesis de los criterios de inclusión y exclusión

Table 1. Summary of the inclusion and exclusion criteria

\begin{tabular}{|c|c|}
\hline Criterio & Descripción \\
\hline \multirow{7}{*}{ Inclusión } & $\begin{array}{l}\text { Procedimientos agiles y flexibles, que permitan la } \\
\text { modificación de cada uno de los entregables si es necesario }\end{array}$ \\
\hline & $\begin{array}{l}\text { Herramientas que promovieran la comunicación abierta entre } \\
\text { los individuos }\end{array}$ \\
\hline & Adaptabilidad al constante cambio \\
\hline & Minimización del riesgo \\
\hline & Optimización de los costos, evitando reprocesos \\
\hline & Toma de decisiones sencillas y resolución de conflictos eficaz \\
\hline & Altas tazas de interactividad entre los equipos \\
\hline \multirow{5}{*}{ Exclusión } & El cliente no sea parte del desarrollo del proyecto \\
\hline & $\begin{array}{l}\text { Exista una dependencia de los equipos frente a una figura de } \\
\text { liderazgo o guía }\end{array}$ \\
\hline & $\begin{array}{l}\text { La metodología a usar no se adapte a grandes aumentos de la } \\
\text { demanda }\end{array}$ \\
\hline & $\begin{array}{l}\text { La metodología solo brinde soluciones específicas y no } \\
\text { generales }\end{array}$ \\
\hline & Su proceso productivo sea lineal \\
\hline
\end{tabular}

La herramienta debió ser versátil, si esta no tenía un componente multidisciplinar no era válida para el ejercicio

Fuente: elaboración propia.

En cuanto a gestión del conocimiento (gc), se analiza la gestión de equipos virtuales y uno de los más importantes, pues se considera que la interacción entre los individuos y el uso de las herramientas propuestas gestan la cocreación de nuevos saberes, pues al garantizar la articulación de participantes con competencias y capacidades diferentes, enriquece el proceso de aprendizaje en el marco de la ejecución de los proyectos (Mclver et al., 2015). Adicionalmente, las experiencias pasadas juegan un rol importante, ya que dejan una serie de lecciones aprendidas y oportunidades de mejora que pueden ser compartidas con cada uno de los miembros del equipo; así, se puede evitar caer en los mismos errores (Riesco, 2006). A continuación, se muestra el análisis de cada uno de los modelos.

En el modelo de Navigator de Skandia se relacionan de forma cíclica las finanzas, clientes, procesos, personas, renovación y desarrollo, evidenciando la importancia de medir cuantitativamente el capital intelectual en las compañías. Esto ha tenido una relevancia significativa, debido a que se pensó en el auge de la economía basada en el conocimiento; por tal motivo, este modelo permite difundir el tema en los entornos empresariales y académicos de una manera sencilla y maleable (Edvinsson \& 
Malone, 1999). En la herramienta de evaluación del conocimiento kmat descansan cuatro pilares fundamentales: tecnología, cultura, liderazgo y medición, que enfatiza en la forma en que las compañías capacitan a las personas para una comunicación asertiva y efectiva (Ojeda et al., 2013).

El modelo seci acopla el conocimiento tácito y el explícito. Surge de la interacción de sus distintos ejes de acción, puesto que pone en contexto los saberes que se adquieren a través de la experiencia y por medio de una interacción documentada se transforman en know-how replicables para las compañías, validados por buenas prácticas organizacionales (Nonaka \& Takeuchi, 1995).

Finalmente, el modelo kpmg está basado en un enfoque sistémico organizacional, en el que prima el aprendizaje de las personas a través de su cultura, liderazgo y estrategias en los sistemas de información (Rachinger et al., 2019). En razón a estos, se plantearon unos criterios de inclusión generales que sirvieran como filtro para la selección de herramientas útiles en la conservación y generación de conocimientos, tal como se observa en la Tabla 2.

Tabla 2. Criterios de inclusión

Table 2. Inclusion criteria

\begin{tabular}{|c|c|}
\hline Variable & Descripción \\
\hline \multirow{4}{*}{ Impacto } & Aprendizaje continuo \\
\hline & Cultura organizacional \\
\hline & $\begin{array}{l}\text { Conversión, almacenamiento, y administración del } \\
\text { conocimiento }\end{array}$ \\
\hline & $\begin{array}{l}\text { Distribución del tema del capital intelectual en } \\
\text { ambientes empresariales y académicos }\end{array}$ \\
\hline \multirow{4}{*}{ Estrategia } & $\begin{array}{l}\text { Las herramientas deben tener una visión } \\
\text { sistemática u holística }\end{array}$ \\
\hline & El conocimiento debe ser una estrategia \\
\hline & $\begin{array}{l}\text { Interacción entre los individuos para el intercambio } \\
\text { de conceptos }\end{array}$ \\
\hline & Capacidad de trabajo colaborativo \\
\hline \multirow{2}{*}{ Frecuencia } & $\begin{array}{l}\text { Repetición de los conceptos a lo largo de los } \\
\text { modelos y herramientas analizadas }\end{array}$ \\
\hline & Relevancia y pertinencia de los conceptos \\
\hline
\end{tabular}

Fuente: elaboración propia.

En el proceso se identificaron los factores que interrumpen el flujo exitoso de los equipos virtuales, información que se seleccionó a raíz del análisis de todas las herramientas utilizadas en este modelo y representadas en la Figura 1. 


\begin{tabular}{|l|c|}
\hline Factor 1 & $\left.\begin{array}{c}\text { Conformación del equipo. } \\
\text { Factor 2 } \\
\text { Factor 3 } \\
\text { Factor } 4 \\
\text { Factor } 5\end{array}\right]$ Tiempo. \\
Comunicación. \\
Redes de conexión virtual.
\end{tabular}

Figura 1. Factores que interrumpen el flujo exitoso de los equipos virtuales. Figure 1. Factors that interfere with the successful flow of virtual machines Fuente: elaboración propia.

En consecuencia, de las herramientas planteadas en la sección anterior y bajo los análisis hechos entre instrumentos, y contando con los criterios de inclusión y exclusión en cada una de las aristas, el resultado fue la creación del modelo de gestión de equipos virtuales en el que se tuvieron cuenta los criterios expuestos en la Tabla 3.

Tabla 3. Criterios para la creación del modelo de gestión de equipos virtuales Table 3. Criteria for creating the virtual team management model

\begin{tabular}{|c|c|}
\hline \multirow{6}{*}{$\begin{array}{c}\text { Criterios para la } \\
\text { creación del }\end{array}$} & Pertinencia de los conceptos \\
\hline & Relevancia de los conceptos \\
\hline & Repetitividad de los conceptos entre los modelos herramientas \\
\hline & Conversión del conocimiento \\
\hline & Estructuración de los proyectos, generando un panorama holístico del mismo \\
\hline & Aprendizaje continuo en cada uno de los eventos \\
\hline mode & Adaptabilidad al entorno \\
\hline gestión de & Herramientas que permitieran tener comunicación fluida y abierta \\
\hline equipos & Reducción y minimización de los riesgos \\
\hline virtuales & Modelos que especifican claramente los roles del equipo \\
\hline & Toma de decisiones colaborativamente \\
\hline & Conformación de grupos multidisciplinarios \\
\hline & Plataformas que permitieran virtualizar el proceso \\
\hline
\end{tabular}


En este sentido, las herramientas, elementos y estrategias que más se justaron a los criterios de inclusión para el modelo de gestión de equipos virtuales en la ejecución de proyectos académicos colaborativos fueron las personas, como se puede observar en los modelos de gestión del conocimiento kpmg, Navigartor de Skandia, en las metodologías de gerencia de proyectos como pmbok, Scrum y, finalmente, en los roles de equipo del doctor Belbin. Las personas son el núcleo principal para que un sistema funcione y se desarrolle, por tal motivo el modelo en que estas fueron elaboradas pertenece al primer nivel.

La segunda variable, más representativa en los modelos y metodologías analizadas, fueron los procesos, pues el Scrum, pmbok, seci y kmat develaron la relevancia de los procesos internos y externos que se ejecutaban en los proyectos a distancia. Cada uno de estos conceptos mostró el enfoque particular que se debía considerar en el momento de liderar proyectos, como se expone en la Tabla 4.

Tabla 4. Procesos para considerar en el liderazgo de proyectos Table 4. Processes to consider in project leadership

\begin{tabular}{|c|c|}
\hline Procesos & Descripción \\
\hline Ágiles & $\begin{array}{l}\text { Flujos y metodologías que permitan maximizar los } \\
\text { recursos sin afectar el proceso del proyecto. }\end{array}$ \\
\hline \multirow{3}{*}{ Estrictos } & Mecanismos que permitan ser evaluados. \\
\hline & Mecanismos que permitan ser controlados. \\
\hline & Mecanismos que permitan ser estructurados \\
\hline \multirow[b]{2}{*}{ Knowledge management } & $\begin{array}{l}\text { Procesos basados en la conversión del } \\
\text { conocimiento. }\end{array}$ \\
\hline & $\begin{array}{l}\text { Procesos donde se identifique, almacene, difunda y } \\
\text { se cree información }\end{array}$ \\
\hline
\end{tabular}

Fuente: elaboración propia.

En $\mathrm{SECl}$ se evidenció que al inicio de cada proyecto virtual existe un conocimiento tácito que, al someterse a un proceso, se convierte en explícito, y se puede usar como punto de partida. En consecuencia, se incluye el método SECl en el modelo propuesto, pues este ayuda a transformar las experiencias pasadas en documentos base para futuras interacciones, identificando, seleccionando, almacenando y difundiendo el conocimiento (Nonaka \& Takeuchi, 1995).

En la tecnología se observó una fuerte pertinencia del uso de las tecnologías en ambientes virtuales, pues, si se quiere perpetuar la información y generar proyectos flexibles, esta es una buena opción; el modelo KMAT, Scrum y las TICs, así lo demuestran, pues, en sus principales variables, en aquellas que enmarcan cada uno de estos modelos y herramientas, hubo presencia de tecnología, como la plataforma Monday, Drive y Skype. De tal modo, el mayor resultado obtenido en esta investigación es la generación del modelo que se presenta en la Figura 2, llamado Sistema Escalonado de Gestión de Equipos Virtuales (Segev). 


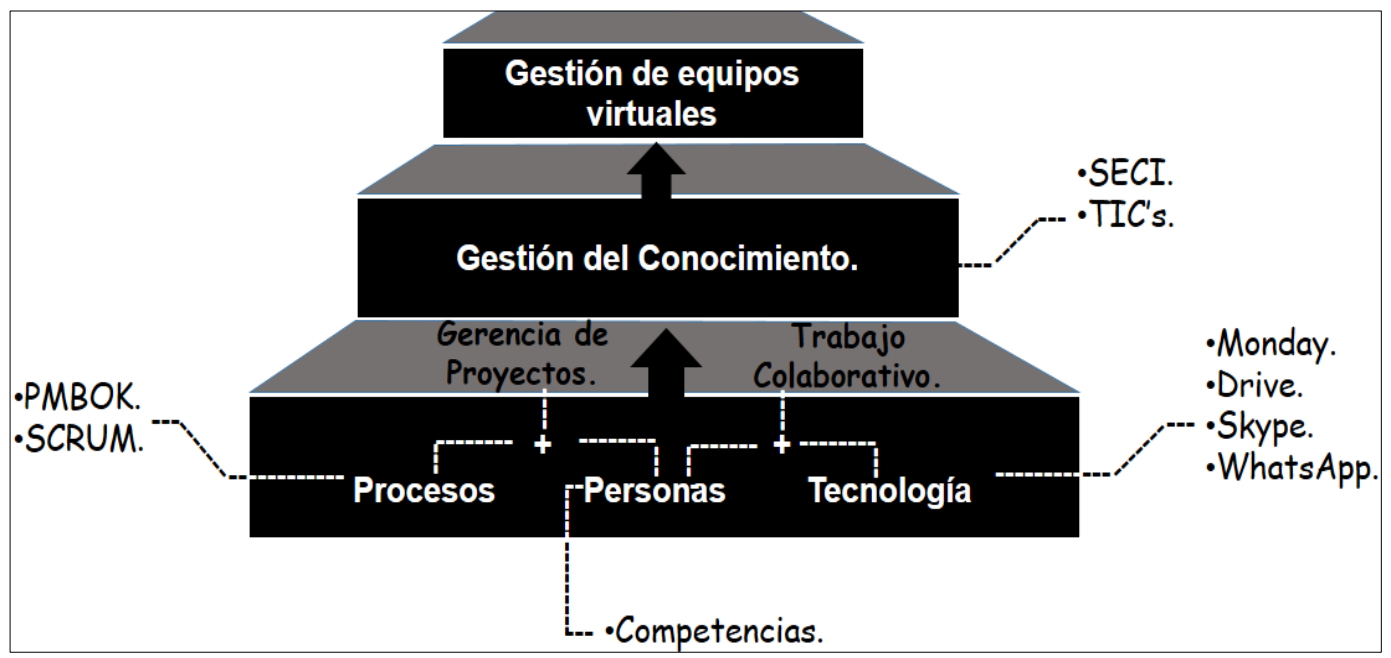

Figura 2. Sistema Escalonado de Gestión de equipos virtuales (Segev)

Figure 2. Tiered system for virtual team management Fuente: elaboración propia.

Dicho modelo se dividió en tres niveles. En el primer nivel se pretende identificar preguntas como qué, quién y cómo, pues, al determinar estos tres aspectos, se genera un panorama holístico de los actores que intervienen en un modelo de gestión de equipos virtuales: las personas, quienes generan el quién, que son las responsables de gestar nuevos conocimientos, conformar equipos de alto desempeño, manipular las plataformas interactivas en los espacios virtuales. Finalmente, también se les atribuye el compromiso de estructurar y trazar el camino para el desarrollo de proyectos a distancia.

Los procesos son elementos que se articulan en el modelo bajo la conjetura del cómo se iba a ejecutar el proyecto, cuáles serían las directrices generales y las variables que se debían contemplar para garantizar un panorama holístico controlado del mismo, utilizando metodologías ágiles que se adapten al constante cambio del entorno, manteniendo una excelente comunicación con el cliente. Asimismo, la tecnología permite dar un rol global e interactivo en el liderazgo de proyectos que se gestan en diferentes posiciones geográficas, pues esta variable flexibiliza y mantiene en contacto cada uno de los actores, dinamizando las actividades integrales de los proyectos y respondiendo de este modo al qué debo utilizar para asegurar que se ejercerá un trabajo colaborativo en el que se garantice el buen uso de las TIC.

Una vez se definieron los elementos base para la dirección de equipos virtuales, se procede a ver la relación que existe entre ellos. Estos datos indicaron que la combinación entre Personas y Procesos da como resultado la Gerencia de Proyectos, pues se debió tener en cuenta un plan de riesgos, calidad, comunicaciones, tiempo y costos, fijándose en el alcance y la interacción entre las áreas del conocimiento, además de un mapeo y adición de micro objetivos que aseguren el cumplimiento de cada uno de estos planes.

De igual forma ocurre con la composición de Personas + Tecnología = Trabajo Colaborativo, gracias a que la conformación de equipos de alto desempeño permitió la retroalimentación de conocimientos 
desconocidos para cada uno de los miembros, potencializando sus fortalezas y mejorando sus debilidades.

En el segundo nivel se encuentra la gestión del conocimiento, que es directamente proporcional a la combinación de Personas + Tecnología + Procesos, ya que la mezcla de estos tres elementos reprodujo nuevos saberes, producto de una interacción de los individuos, quienes compartieron aspectos culturales, sociales y profesionales que alimentan la cocreación e innovación en la gerencia de equipos virtuales basados en la excelente manipulación de las TIC. Así, el paso a seguir es la identificación, almacenamiento, divulgación y uso de la información.

En el tercer nivel está la gestión de equipos virtuales. Este es el resultado global del uso de gestión del conocimiento, la gerencia de proyectos y el trabajo colaborativo. Estos tres elementos permitieron desarrollar, liderar, estructurar, comunicar y compartir conocimientos o saberes que nutren y naturalizan las dinámicas a distancia. Esto responde a la necesidad del mercado actual, que cada vez más se comporta como un sistema de red donde cada nodo, cliente-empresa, trasmite información entre sí.

\section{DISCUSIÓN}

Se concuerda con la investigación hecha por Journal of Economics en que uno de los principales desafíos que se presentan en estos ejercicios es la coordinación de los equipos a distancia y el empalme que debe existir entre los individuos para generar un entorno agradable de trabajo (Olivos Rossini et al., 2015). Si vemos esta declaración en los resultados obtenidos, se prevé la implementación de dinámicas que mitiguen estas variables y así garantizar la correcta ejecución de los proyectos.

El empleo futuro deberá ser una combinación de habilidades blandas y duras como estrategia de la fuerza laboral para la cuarta revolución industrial (World Economic Forum, 2016). En este sentido, la afirmación de la revista sirve como argumento para apoyar la implementación de las metodologías colaborativas en un contexto de proyectos académicos virtuales, gracias a que ayuda a potenciar, fortalecer y difundir los conocimientos y técnicas en un contexto determinado. Esto genera un impacto positivo en la organización, puesto que se da el espacio para crear equipos de alto rendimiento capaces de reducir la ventaja de sus competidores.

El modelo propuesto se ajusta a la necesidad de desarrollar proyectos académicos colaborativos, es por ello que las herramientas y metodologías incorporadas permiten tener un discernimiento total y parcial del proceso que se ejecutan. Cabe resaltar, como bien lo dicen (Córdoba Cárdenas et al., 2015), no existe un modelo perfecto en la gestión de equipos virtuales, pues en la actualidad, los requerimientos y necesidades de los clientes cambian constantemente gracias a la competitividad global y local del mercado.

Estos hallazgos derivados del análisis de la literatura permiten subsanar las fallas que interrumpían el flujo exitoso de los equipos virtuales como el tiempo reducido, la falta de comunicación, las redes de contacto virtual escasas y de baja calidad, entre otros. Cuando se tiene una respuesta directa por metodologías, como en el caso de los nueve roles de equipo del doctor Belbin, se ayuda a generar un 
ambiente de trabajo agradable, pues se conforman equipos de alto desempeño, lo que quiere decir que se reúne un conjunto de competencias que abarcan todos los puntos de un proyecto, respondiendo entonces a una combinación de habilidades duras y blandas inmersas en un mismo contexto. Adicionalmente, las metodologías ágiles y tradicionales permiten que exista un entorno flexible y comunicativo, reduciendo los tiempos de proceso y riesgos del proyecto, generando un aumento de la rentabilidad y calidad del mismo (Amaro Calderón \& Valverde Rebaza, 2007).

Expertos afirman que a través de la vinculación de las prácticas ágiles en los proyectos se pueden evitar los problemas comunes en ellos, pues aseguran que la vinculación del cliente a las fases del proyecto crea valor en ellos y los faculta para responder a la vulnerabilidad de cambio del entorno (Torres Carreño, 2016). Este es un hallazgo válido para implementar, porque, al generar una comunicación abierta y amigable con el cliente, mostrándole el avance del producto, este tiene la posibilidad de personalizar su producto o servicio según sus gustos. Adicionalmente, la implementación de metodologías como Scrum garantiza la reducción de los riesgos, debido a la supervisión diaria y continua de las actividades.

Como afirman Orengo et al., (2011), es de vital importancia que el trabajo colaborativo en la gestión de equipos a través de la implementación de las TIC en las compañías sea un proceso normal y natural de todas las empresas, pues, gracias a este y la interacción entre los individuos, se gestan nuevos conocimientos y saberes útiles para las organizaciones. A esto se debe que el modelo Segev lo evidencie y respalde en el segundo nivel operativo.

Los criterios de inclusión y exclusión identificados a través del análisis de los modelos de gestión del conocimiento indican que es necesario convertir el conocimiento en un activo importante en las organizaciones, pues en la actualidad este es el que genera una ventaja competitiva, gracias al trabajo colaborativo que se gesta en los equipos de proyectos. Por tal motivo, es útil incorporar estas temáticas en las universidades, para crear un hábito de aprendizaje continuo y autónomo de los individuos (Sánchez Medina, et al., 2007; Rostro Hernández \& Solís Hernández, 2015).

Finalmente, se puede percibir ampliamente que el desarrollo del modelo Segev agrega valor en los proyectos académicos colaborativos, en la medida en que permite obtener un acercamiento con otras culturas, implementando metodologías actuales y de gran impacto en la industria moderna. En este orden de ideas, facilita a quien utilice este modelo la potenciación de habilidades duras y blandas en el marco de la gerencia de proyectos, la gestión del conocimiento y el trabajo colaborativo. Sin embargo, este adelanto tecnológico y científico sirve como primer insumo y punto de partida para la cocreación en ambientes académicos, donde, por tener un componente flexible, se permite la vinculación de otras herramientas ingenieriles y de tendencia mundial, lo que lleva al lector a pensar sistémicamente en la meálvareora continua, una habilidad analítica de gran importancia en era del conocimiento.

\section{CONCLUSIONES}

A raíz de los resultados obtenidos para la generación del modelo de gestión de equipos, es necesario vincular las metodologías ágiles para liderar los proyectos en ambientes virtuales, como en este caso Scrum, pues esta es dinámica y flexible, se acopla al desarrollo natural del ejercicio, minimiza los riesgos y maximiza la oportunidad de culminar el proyecto exitosamente. 
Gracias a la literatura analizada, se puede concluir que el modelo debe poseer una sección en la que transfiera el conocimiento, lo almacene y lo divulgue a todos los involucrados en el proyecto. Por tal motivo, Segev implementa el modelo de gestión del conocimiento en espiral seci, con el que garantiza transformar todos los aprendizajes tácitos en explícitos.

Como se evidencia en los resultados, es de vital importancia que el modelo de gestión de equipos virtuales esté pensado para cubrir las variables de ambiente de equipo, tiempo, comunicación y redes de conexión virtual; sin estas, el modelo tenderá a fracasar, pues son las mínimas aristas que debe brindar el modelo. Cada una de las herramientas utilizadas en el modelo están bajo el pilar fundamental de tener una visión sistémica, pues si no se garantiza esta cualidad, el modelo no se comportaría como un nodo y no generaría conexiones con otros campos del mismo, resultado del análisis y selección de las variables evidenciadas en el cuerpo documento.

Esta investigación permitió responder la pregunta problema. Se pudieron identificar los elementos y metodologías necesarias para la gestión de equipos virtuales en la ejecución de proyectos académicos colaborativos, las cuales permiten generar mejores resultados, debido a que tienen como prioridad la reducción de riesgos y tiempo con un énfasis en las fases de los proyectos mediado por técnicas cooperativas que posibilitan la cocreación de nuevos saberes útiles en la implementación de este tipo de ejercicios.

El desarrollo de un modelo de gestión de equipos permite tener una visión holística de la dinámica operativa de los proyectos a distancia, pues en estos se presentan una serie de herramientas y técnicas que agilizan y flexibilizan el ejercicio. Esto no quiere decir que exista un modelo perfecto, pues al tener en cuenta la variable cambio del entorno, automáticamente es necesario ajustar el modelo a los requerimientos del proyecto. La selección de los individuos antes de comenzar un proyecto resulta exitosa, pues se pueden conocer las competencias que presenta el personal, clasificándolas según sus habilidades; de este modo, se puede generar e identificar el impacto en el desarrollo organizacional del mismo.

A raíz del modelo propuesto, se puede concluir que la interacción entre personas, procesos y tecnología gesta nuevos conocimientos que tienen una influencia directamente proporcional en la ejecución de proyectos a distancia, pues, al estar inmerso en un entorno negativo, la gestión de equipos se tornará un poco complicada, dificultando el resultado final del producto o servicio elaborado.

Los resultados arrojados por la literatura demuestran que, en los equipos virtuales, prima el buen uso de las TIC, ya que, sin una buena gestión de estas, sería difícil la realización de los proyectos, dado que la identificación, selección, almacenamiento, uso y difusión de la información no sería objetiva, por lo que se verían distorsionados los procesos de comunicación entre los pares. Se puede concluir también que la conformación de un equipo basado en el trabajo colaborativo tiene un efecto positivo en los estudiantes, ya que los transforma en miembros activos en su proceso de aprendizaje mediante la interacción con otras personas, quienes tienen competencias diferentes, potencializando sus habilidades y fortaleciendo sus debilidades.

La conversión del conocimiento resulta muy práctica, puesto que, pasar de un conocimiento tácito a uno explícito, significa no arrancar los proyectos desde cero, sino que ya existe un punto de partida 
y es de gran importancia en la gestión de equipos virtuales. Los proyectos deben tener un énfasis en la gerencia predictiva, respondiendo a necesidades de tiempo, costos, riesgos y flexibilidad, pues el mercado es demasiado susceptible al cambio, por lo que se debe de garantizar que el proyecto no fracasará. Por tanto, la gestión del conocimiento permite definir el alcance del proyecto basado en las capacidades y cualidades de sus miembros e infraestructura local.

Es natural saber que existe una ruptura entre lo teórico y lo práctico, y de esta idea se evidencia una brecha gigante entre estos dos campos. En razón a esto, es importante seguir implementando estas dinámicas en las instituciones para fortalecer la gestión de cambio que puedan impartir los profesionales en formación, subsanando los vacíos que hoy existen en el campo educativo latinoamericano. Para lograr esto los estudiantes deben apoyar y proponer nuevos ejercicios que permitan el intercambio cultural y cognitivo entre las mismas regiones.

Finalmente, este modelo ayuda a mitigar el impacto del rápido cambio del entorno, que, de hecho, ya ha afectado a las instituciones educativas. Este modelo sirve como estrategia para ser implementada en los cursos y generar una mayor conciencia y captación de saberes, que, usados de la mejor manera, repercutirán en la creación de nuevos conocimientos aplicables para cada una de las partes involucradas.

\section{REFERENCIAS}

Acevedo-Correa, Y.; Aristizábal-Botero, C. A.; Valencia-Arias, A.; Bran-Piedrahita, L. (2020). Formulación de modelos de gestión del conocimiento aplicados al contexto de instituciones de educación superior. Información tecnológica, v. 31, n. 1, 103-112. http://dx.doi.org/10.4067/S0718-07642020000100103

Acevedo-Prins, N. M.; Jiménez-Gómez, L. M. (2015). Índice para la medición de la competitividad en Colombia. Revista CEA, v. 1, n. 2, 109-121. https://doi.org/10.22430/24223182.136

Addati, G. A. (2017). Equipos virtuales de trabajo. URL

Amaro Calderón, S. D.; Valverde Rebaza, J. C. (2007). Metodologías ágiles. Universidad Nacional de Trujillo.

Ángel Uribe, I. C.; Cano Vásquez, L. M. (2011). Experience of a collaborative work with students and teachers of diverse latitudes mediated by communication and information technologies: Collaborative interuniversity project, Colombian chapter. Revista Q: Educación Comunicación Tecnología, v. 6, n. 11. URL

Antunes, H. J. G.; Gonçalves Pinheiro, P. (2019). Linking knowledge management, organizational learning and memory. Journal of Innovation \& Knowledge, v. 5, n. 2, 140-149. https://doi.org/10.1016/j.jik.2019.04.002

Apolo Peñaloza, E. Y.; Hidalgo Ochoa, A. N. (2016). El trabajo colaborativo como estrategia para fortalecer la solidaridad y autonomía de los estudiantes. URL 
Barros, M. V.; Ferreira, M. B.; do Prado, G. F.; Piekarski, C. M.; Picinin, C. T. (2020). The interaction between knowledge management and technology transfer: a current literature review between 2013 and 2018. The Journal of Technology Transfer, 1-22. https://doi.org/10.1007/s10961-020-09782-w

Bates, A. W. T.; Sangra, A. (2012). La gestión de la tecnología en la educación superior. Editorial Octaedro.

Beck, K. (1999). Extreme Programming Explained: Embrace Change. Addison Wesley Longman.

Belbin. (2014). Cómo utilizar los Informes Belbin para mejorar el autoconocimiento y aumentar la eficacia personal. $\underline{U R L}$

Belbin. (2015). La guía definitiva para conocer los Roles de Equipo Belbin. URL

Cavaller, V.; Sánchez-Añón, S.; Codina, L.; Pedraza, R. (2014). Estrategias y gestión de la comunicación online y offline. Editorial UOC.

Córdova Cárdenas, G. M.; Palacio Cinco, R. R.; Cortez González, J. (2015). Desempeño del trabajo en equipo y plataformas virtuales educativas. Revista OPCIÓN, v. 31, n. 5, 262-277. URL

Cuatrecasas Arbós, L. (2012). Gestión de proyectos: Producción por puestos fijos metodología pmbok.

De la Cruz Lablanca, I. (2015). Apoyo administrativo a la gestión de recursos humanos. Aula Mentor. $\underline{U R L}$

Edvinsson, L; Malone, M. (1999). Capital Intelectual. cómo identificar y calcular el valor de los recursos intangibles de su empresa. Gestión 2000.

Estrada Sentí, V. (2014). Decisiones inteligentes: herramientas y metodologías (curso 17). URL

Gilibets, L. (2013). Qué es la metodología Kanban y cómo se utiliza. URL

Gupta, S.; Gallear, D.; Rudd, J.; Foroudi, P. (2020). The impact of brand value on brand competitiveness. Journal of Business Research, v. 112, 210-222. https://doi.org/10.1016/j.jbusres.2020.02.033

Guitert, M.; Giménez, F. (2000). Trabajo cooperativo en entornos virtuales de aprendizaje. En Duart, J. M.; Sangrá, A. Aprender en la Virtualidad. Gedisa.

Hernández Cotón, S.; Sánchez Gutiérrez, J. (2006). Modelo conceptual de las variables a investigar en el desarrollo de la gestión del conocimiento en las empresas. Mercados y Negocios, n.13, 38-60. $\underline{U R L}$ 
Jasso Villazul, J.; Torres Vargas, A. (2008). La gestión del conocimiento en las empresas y organizaciones: el dilema de la absorción, creación, resguardo y aprendizaje. Revue Sciences de Gestion, n. 66, 258-302. URL

Lui, G.; Lavazza, L.; Tosi, D. (2020). Evolution of functional size measures through ICONIX process phases. Journal of Software: Evolution and Process, v. 32, n. 5, e2240. https://doi.org/10.1002/smr.2240

Martínez Villalobos, G.; Arciniegas, A. M.; Lugo González, C. A. (2016). Formación docente en TIC con el Centro de Innovación Educativa CIER-SUR. Trilogía Ciencia Tecnología Sociedad, v. 8, n. 14, 65-80. https://doi.org/10.22430/21457778.417

Mclver, D.; Fitzsimmons, S.; Flanagan, D. (2015). Instructional Design as Knowledge Management: A Knowledge-in-Practice Approach to Choosing Instructional Methods. Journal of Management Education, v. 40, n. 1, 47-75. https://doi.org/10.1177/1052562915587583

Mora-Vicarioli, F.; Hooper-Simpson, C. (2016). Trabajo colaborativo en ambientes virtuales de aprendizaje: Algunas reflexiones y perspectivas estudiantiles. Revista Electrónica Educare, v. 20, n. 2, 1-26. https://doi.org/10.15359/ree.20-2.19

Nonaka, I.; Takeuchi, H. (1995). The Knowledge-Creating Company: How Japanese companies create the dynamics for innovation. Oxford University Press.

Ojeda, M.; Revette, K.; Rodríguez, V. (2013). Modelo de gestión del conocimiento kmat. Universidad Privada Rafael Belloso. URL

Olivos Rossini, M.; Rincón, S.; Rutkowski, A.-F. (2015). The Link Class Project: Collaborative virtual teams between Peru and The Netherlands. Journal of Economics, Finance and Administrative Science, v. 20, n. 39, 137-140. https://doi.org/10.1016/j.jefas.2015.10.001

Orengo, V.; Zornoza, A.; Peiró, J. M. (2011). Gestión de equipos virtuales en las organizaciones laborales: algunas aportaciones desde la investigación. Papeles del psicólogo, v. 32, n. 1, 82-93. $\underline{U R L}$

Peña, M. M. (2016). La importancia del Trabajo Colaborativo en la enseñanza de la Metodología de Diseño de Proyectos, In: XX Congreso de la Sociedad Iberoamericana de Gráfica Digital. Blucher Design Proceedings, v.3 n.1, 175-179. https://doi.org/10.5151/despro-sigradi2016-414

Pérez Porto, J.; Merino, M. (2016). Definiciones de Compeitividad. $\underline{\text { URL }}$

Pinzón Rincon, J.; Remolina Millán, A. (2017). Evaluation of tools for construction projects management based on PMI fundamentals and experience. Prospectiva, v. 15, n. 2, 51-59. URL

Podestá, P. (2014). El trabajo colaborativo entre docentes: experiencias en la Especialización Docente Superior en Educación y tic. Congreso Iberoamericano de Ciencia, Tecnología, Innovación y Educación. Buenos Aires. 
Rachinger, M.; Rauter, R.; Müller, C.; Vorraber, W.; Schirgi, E. (2019), Digitalization and its influence on business model innovation. Journal of Manufacturing Technology Management, v. 30, n. 8, 1143-1160. https://doi.org/10.1108/JMTM-01-2018-0020

Riesco, G. M. (2006). El negocio es el conocimiento. Diaz de Santos.

Rostro Hernández, P. E.; Solís Hernández, O. (2015). Conceptualizando el diálogo entre cultura y aprendizaje organizacional. Trilogía Ciencia Tecnología Sociedad, v. 7, n. 12, 95-103. https://doi.org/10.22430/21457778.490

Rugeles Contreras, P. A.; Mora Gonzales, B.; Metaute Paniagua, P. M. (2015). El rol del estudiante en los ambientes educativos mediados por las TIC. Revista Lasallista de Investigación, v. 12. n. 2, 132-138. https://doi.org/10.22507/rli.v12n2a14

Sánchez Medina, A. J.; Melián Gonzáles, A.; Hormiga Pérez, E. (2007). El concepto de capital intelectual y sus dimensiones. Investigaciones Europeas de Dirección y Economía de la Empresa. v. 13, n. 2, 97-111. URL

Sung, S. Y.; Choi, J. N. (2012). Effects of team knowledge management on the creativity and financial performance of organizational teams. Organizational Behavior and Human Decision Processes, v. 118, n. 1, 4-13. https://doi.org/10.1016/j.obhdp.2012.01.001

Teagarden, M. B. (2020). Global talent management: The foundation of global competitiveness. Thunderbird International Business Review, v. 62, n. 4, 327-328. https://doi.org/10.1002/tie.22141

Torres Carreño, A. P. (2016). Características de las metodologías ágiles de gestión de proyectos y su aplicación en las organizaciones matriciales. Universidad Nacional Abierta y a Distancia.

Vailant, D. (2016). Trabajo colaborativo y nuevos escenarios para el desarrollo profesional docente. Revista Docencia, v. 60, 7-13.

World Economic Forum. (2016). The Future of Jobs Employment, Skills and Workforce Strategy for the Fourth Industrial Revolution. URL 